\title{
Concomitant Transapical Transcatheter Aortic Valve Replacement and Transapical Balloon Mitral Valvuloplasty to Treat Severe Aortic Stenosis with Severe Mitral Stenosis
}

\author{
Peijian Wei, MD, ${ }^{1,2}$ Jian Liu, $M D,{ }^{1}$ Huiming Guo, $\mathrm{MD}, \mathrm{PhD}^{1}$ \\ ${ }^{1}$ Department of Cardiovascular Surgery, Guangdong Provincial Key Laboratory of South China Structural Heart Disease, \\ Guangdong Cardiovascular Institute, Guangdong Provincial People's Hospital (Guangdong Academy of Medical Sciences), \\ Guangzhou, China; ${ }^{2}$ Shantou University Medical College, Shantou, China
}

\section{ABSTRACT}

This paper reports concomitant transapical transcatheter aortic valve replacement (TA-TAVR) and transapical balloon mitral valvuloplasty (TA-BMV) for the first time. A 72-yearold man with a diagnosis of rheumatic severe aortic stenosis with mild insufficiency and rheumatic severe mitral stenosis with mild insufficiency was referred to the Department of Cardiac Surgery of Guangdong Provincial People's Hospital. After the interdisciplinary discussion in the heart team (cardiac surgeon, cardiologist, anesthesiologist and image specialist), we decided to perform concomitant TAVR and BMV through one transapical approach considering the patient's preference, NYHA class IV heart failure, and the calculated perioperative risk (Euroscore II 3.74\%, STS score for the combined mitral and aortic procedure is not available). No intraoperative or postoperative complications were observed.

\section{INTRODUCTION}

Over the recent years, transcatheter aortic valve replacement (TAVR) has become an established procedure alternative for patients with inoperable or high-risk symptomatic aortic stenosis, and the feasibility and safety of the transapical TAVR (TA-TAVR) system J-valveTM for treating high-risk patients with serve aortic disease has been proved [Zhu 2017]. As for symptomatic patients with severe mitral

Received March 30, 2021; accepted May 24, 2021.

Correspondence: Hui-Ming Guo, Department of Cardiovascular Surgery, Guangdong Provincial Key Laboratory of South China Structural Heart Disease, Guangdong Cardiovascular Institute, Guangdong Provincial People's Hospital (Guangdong Academy of Medical Sciences), Guangzhou 510080, China (e-mail: guobuiming@vip.tom.com) and Fian Liu, Department of Cardiovascular Surgery, Guangdong Provincial Key Laboratory of South China Structural Heart Disease, Guangdong Cardiovascular Institute, Guangdong Provincial People's Hospital (Guangdong Academy of Medical Sciences), Guangzhou 510080, China, (e-mail: Fameslau1984@sina.com). stenosis, percutaneous mitral commissurotomy has been recommended, according to 2017 ESC/EACTS guideline for the management of valvular heart disease [Baumgartner 2017]. Patients with multivalvular stenosis comprise a special patient population, which is characterized by high perioperative morbidity and mortality, and simultaneous TATAVR and transapical transcatheter mitral valve replacement (instead of transapical balloon valvuloplasty due to the high Wilkins score) has been reported to treat severe aortic stenosis with severe mitral stenosis [Elkharbotly 2016]. To our knowledge, this paper reports concomitant transapical transcatheter aortic valve replacement (TA-TAVR) and transapical balloon mitral valvuloplasty (TA-BMV) for the first time.

\section{CASE REPORT}

A 72-year-old man complaining of shortness of breath on exertion for about six months, with a New York Heart Association (NYHA) functional class of IV, was referred to our hospital in September 2020. Transthoracic echocardiogram (TTE) revealed a preserved ejection fraction, a severe aortic stenosis (mean pressure gradient $83 \mathrm{mmHg}$, peak velocity $5.8 \mathrm{~m} / \mathrm{s}$ ) with mild insufficiency, and a severe mitral stenosis (opening area $1.3 \mathrm{~cm} 2$ ) with mild insufficiency. Considering the patient's preference, the advanced age, NYHA class IV heart failure, patient frailty, the Wilkins' echo score of 7, the calculated perioperative risk (Euroscore II 3.74\%, STS score for the combined mitral and aortic procedure is not available) and calcified iliacal, we decided to perform TAVR and BMV through one transapical approach after the interdisciplinary discussion in the heart team (cardiac surgery, anesthesiology, ultrasound).

Electrocardiography (ECG) showed sinus rhythm and coronary artery angiography revealed no stenosis. Preoperative evaluation and sizing were performed with further computed tomography angiography of the aorta and coronary artery, which confirmed a tricuspid aortic valve (TAV) with incrassation and severe calcification. The average diameter, area, and perimeter of aortic annuls were $19.3 \mathrm{~mm}, 266.5 \mathrm{~cm} 2$ and $59.9 \mathrm{~mm}$, respectively. The mean diameter of the aortic annulus was $19.3 \mathrm{~mm}$, and the sinus of Valsalva was $23.7 \mathrm{~mm}$ $\times 23.5 \mathrm{~mm} \times 26 \mathrm{~mm}$. The diameter of ascending aorta and sinotubular junction were $30.8 \mathrm{~mm}$ and $19.2 \mathrm{~mm}$. (Figure 1) 




Figure 1. Computed tomography angiography of the aorta and coronary artery. (A) The diameter of aortic annuls; (B, C) The diameter of ascending aorta and sinotubular junction, respectively; (D) The diameter of the sinus of Valsalva.

Ostium heights of the left and right coronary artery were 8.2 $\mathrm{mm}$ and $15.7 \mathrm{~mm}$, and it was suggested that we should be aware of the potential occlusion of left coronary artery during the procedure. The optimal C-arm angulation was anterior and posterior (AP) position, which can display three aortic sinuses on the same level.

In a hybrid operating room, the patient was in the position of supine under general anesthesia with endotracheal intubation, and transesophageal echocardiography (TEE) was performed to further evaluate the anatomic features of the aortic annulus and mitral annulus. Preoperative TEE confirmed a severe aortic stenosis mild insufficiency and severe mitral stenosis with mild insufficiency. Then a temporary endocardial pacing lead was placed in the right ventricle via internal jugular vein. The patient was covered by sterile drapes to expose the full thorax and the groin after the disinfection of skin. A left anterolateral incision $(5 \mathrm{~cm})$ was made in the fifth intercostal space under the left breast and purse-string sutures were placed on the apex to secure the puncture point. Meanwhile, a pig tail catheter was inserted into non-coronary sinus of Valsalva via a femoral artery. A contrast shot was given to visualize the position of three aortic sinus and coronary ostium, calcification of aortic leaflets, and the relationship between aortic annuls and the puncture point. A bolus of $1 \mathrm{mg} / \mathrm{kg}$ dose of heparin was given to achieve activated clotting time (ACT) of $>250 \mathrm{~s}$. When the $\mathrm{C}$-arm was in the position of left anterior oblique and caudal, the location of the apex is determined under the guidance by digital subtraction angiography (DSA). Then a super-stiff guidewire was placed through the aortic valve in the descending aorta after the apical puncture, which is guided by palpation and intraoperative TEE. To distract the calcified and narrow aortic valve, a balloon catheter is placed at the annular level, and balloon valvuloplasty was performed under rapid ventricular pacing (160 times/ minute) with a $20 \mathrm{~mm}$ balloon (NuMED Canada Inc.), while the ventilation is stopped temporarily (Figure 2A). (Figure 2) Thereafter, under fluoroscopic guidance, the delivery system of a second-generation J-valve system (Jie Cheng Medical Technologies, Suzhou, China) was bluntly inserted into the left ventricle and positioned above the aortic valve. The three 'U-shape' claspers were totally released first, and the delivery catheter was gently pulled back until the three claspers were inside the aortic sinus, which was confirmed by both fluoroscopy and TEE (Figure 2B). After that, the crimped and selfexpandable prosthesis valve was brought down to the annular plan with guidance of the claspers (Figure 2C) and deployed 

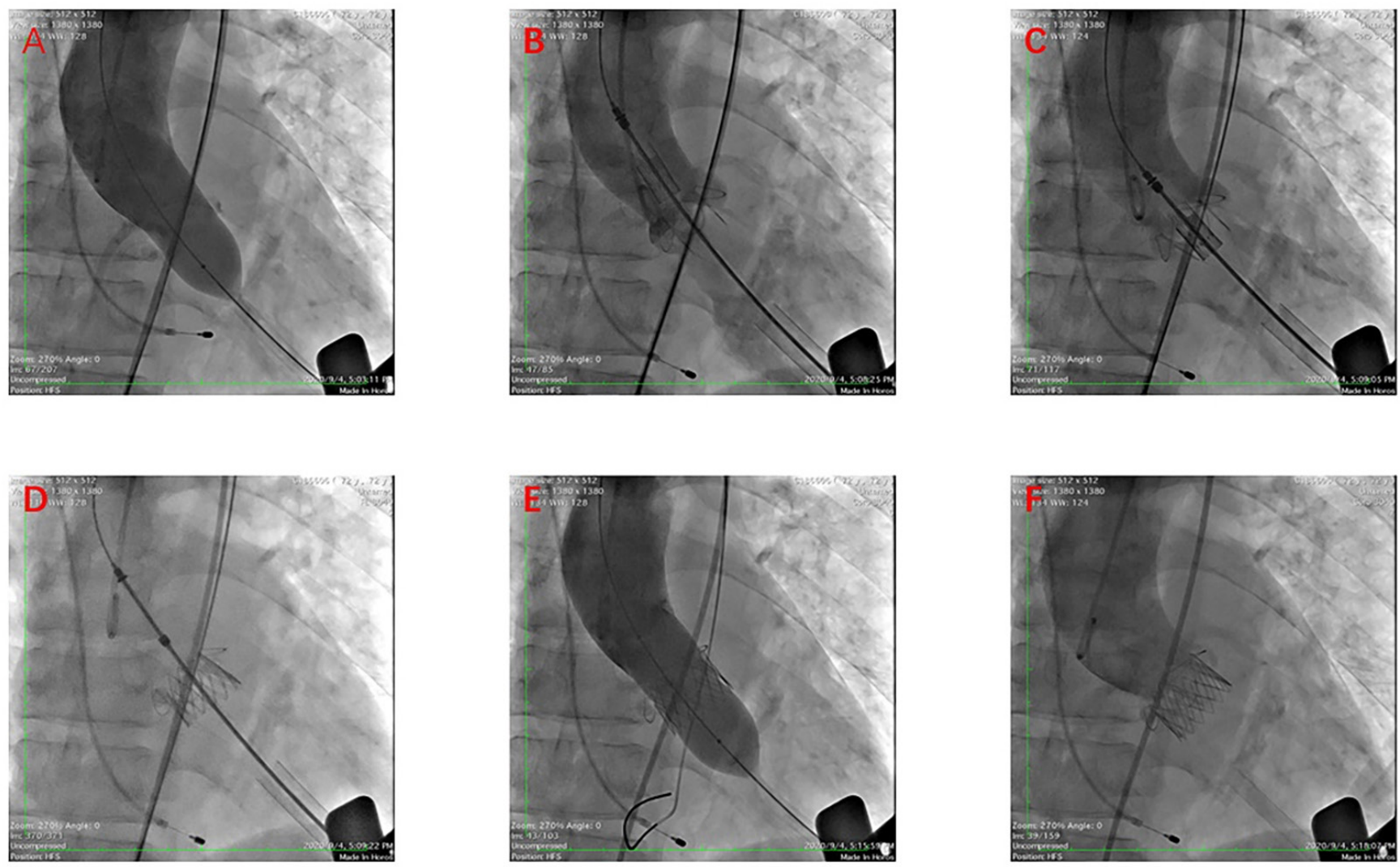

Figure 2. The course of transapical aortic valve replacement. (A) Pre-implantation balloon valvuloplasty using a 20mm balloon; (B) Three 'U-shape' claspers released and were pulled back into the aortic sinus; (C) The prosthesis aortic valve was pulled back to the annular plan; (D) The prosthesis aortic valve was released and expanded to fit the aortic leaflet; (E) Post-implantation balloon valvuloplasty; (F) Angiogram revealed no prosthetic aortic paravalvular leakage.
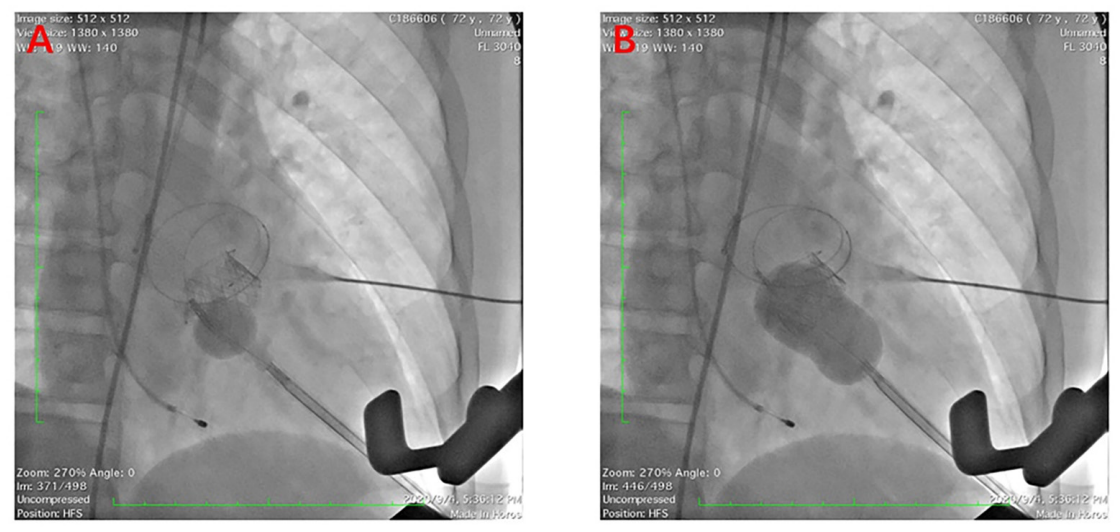

Figure 3. Dilation of the inserted Inoue-balloon for mitral valve under fluoroscopic guidance. (A) The anterior half of the Inoue-balloon was dilated in the left atrium and then pulled back into the plan of mitral annulus; (B) The posterior half of balloon was dilated. 
automatically without rapid ventricular pacing (Figure 2D). Three claspers were further released to fit the native anatomy of the aortic sinus during the valve stent was brought down to the annular position. After retrieval of the delivery system, aortic root angiography and TTE were done to evaluate valve position and function, which revealed a mild-moderate paravalvular leak (aortic velocity $2.8 \mathrm{~m} / \mathrm{s}$ ). We decided to perform a post-implantation dilation using a $20 \mathrm{~mm}$ balloon to make sure the prosthesis valve fit well and deployed fully (Figure $2 \mathrm{E})$. Comparing to the previous status, the prosthesis valve totally deployed to fit the aortic annuls and leaflets after postimplantation balloon valvuloplasty. Angiography confirmed no aortic insufficiency and paravalvular leak with patent coronaries and good prosthesis valve position (Figure 2F). TEE also showed normal function of the J-valve in the good position without paravalvular leak.

Afterward, the delivery system was gently removed, and a $26 \mathrm{~mm}$ Inoue-balloon catheter (Toray Industries, Tokyo, Japan) was introduced into the left ventricle through the same transapical approach. The Inoue-balloon catheter successfully advanced across the mitral valve after numerous attempts. In the next step, the anterior half of the Inoue-balloon was dilated in the left atrium, and it was pulled back into the plan of mitral annulus (Figure 3A) to dilate the posterior half (Figure 3B) under fluoroscopic guidance. (Figure 3) Subsequently, the Inoue catheter was retrieved, and TEE revealed an enlargement of opening area of mitral valve without significant regurgitation. Protamine was given to reverse the heparin. Finally, the hemostasis safely was achieved by tying the purse-string sutures, and the wound was closed by continuous sutures after inserting a chest tube into the pleural space. There were no intra-operative and postoperative complications. The operative time was 135 minutes. The patient was transferred to the intensive unit (ICU) and extubated four hours postoperatively. After an uneventful course at the ICU, the patient was transferred to the general ward on the first postoperative day and was discharged home on day 4. TTE at discharge showed a functional prosthesis aortic valve with a mean gradient of 12 $\mathrm{mmHg}$ (peak velocity $2.4 \mathrm{~m} / \mathrm{s}$ ) and an increased mitral valve (opening area $1.62 \mathrm{~cm} 2$ ). The functional status of the patient improved from NYHA class IV to NYHA class II.

\section{DISCUSSION}

TAVR has been recognized as a safe and effective treatment option for high-risk patients with severe aortic stenosis. As the world population is aging, the high-risk and inoperable elderly patients with a diagnosis of severe aortic stenosis and severe mitral stenosis, comprise a special patient population. Luckily, the indications for TAVR and combined procedure are expanding toward multivalvular disease and even selective low-risk patients thanks to the advancement of device technology, the accrual of the procedural knowledge, and expertise by the operators. And the safety and outcomes in high-risk patients who underwent TA-TAVR by J-valveTM has been proven [Zhu 2018]. Concomitant TA-TAVR and transapical mitral valve repair and simultaneous TA-TAVR and transcatheter mitral valve replacement have already been reported to treat multivalvular disease [Gerosa 2015].

In this case, we decided to perform TAVR and BMV through one transapical approach after discussions with the patient and his family. The reasons are described as follows. First, in 2019, PARTNER 3 trial and Evolut Low Risk Trial showed that TAVR was superior or noninferior to surgical aortic valve replacement at reducing death, stroke, or rehospitalization at one year. So, TAVR is reasonable for this low-risk patient (Euroscore II 3.74\%). Second, percutaneous mitral commissurotomy is recommended as an initial treatment for symptomatic patients without unfavorable characteristics. And the rate of reintervention after percutaneous mitral commissurotomy was $10 \%$ (95\% CI: $8.5 \%$ to $11.7 \%$ ) throughout 23 years, according to a research conducted by MeneguzMoreno. Considering the patient's preference, the advanced age (72 years old), NYHA class IV heart failure, patient frailty, the Wilkins' echo score of 7 and calcified iliacal, TAVR and BMV through one transapical approach was the optimal strategy for this patient. Furthermore, TA-TAVR is the only anterograde TAVR approach and provides easy access to the aortic valve and excellent device controllability. Comparing with other retrograde approaches, the super-stiff guidewire and delivery system can be directed across the valve easily through transapical approach and provide a better coaxiality.

In addition, key to the procedure is the sequence of TATAVR and TA-BMV. Actually, for a pool with obstructed outlet and inlet, it's better to relieve the obstruction of outlet first instead of the inlet. The volume of blood flow would significantly increase after the $\mathrm{BMV}$, and it probably would lead to circulatory collapse if the aortic stenosis was still present. So, we decided to perform TA-TAVR prior to TA-BMV. Besides, the challenge of the procedure is maintaining the position of the prosthesis aortic valve, while performing the balloon valvuloplasty for mitral valve, in case of squeezing out the prosthesis aortic valve. So, we performed the balloon valvuloplasty for mitral valve by injecting quantitative saline under guidance of DSA. No intra-operative and postoperative complications were observed. This provides a new strategy of treatment for multivalvular stenosis, which is feasible and effective.

\section{REFERENCES}

Baumgartner H, Falk V, Bax JJ, et al. 2017. 2017 ESC/EACTS Guidelines for the management of valvular heart disease. Eur Heart J. 38(36):2739-2791.

Elkharbotly A, Delago A, El-Hajjar M. 2016. Simultaneous transapical transcatheter aortic valve replacement and transcatheter mitral valve replacement for native valvular stenosis. Catheter Cardiovasc Interv. 87(7):1347-1351.

Gerosa G, D'Onofrio A, Manzan E, et al. 2015. One-stage off-pump transapical mitral valve repair and aortic valve replacement. Circulation. 131(19): e430-e434.

Zhu L, Guo Y, Wang W, et al. 2018. Transapical transcatheter aortic valve replacement with a novel transcatheter aortic valve replacement system in high-risk patients with severe aortic valve diseases. J Thorac Cardiovasc Surg. 155(2):588-597. 